03,13

\title{
Остановка и разворот дислокаций несоответствия при росте нитрида галлия на подложках $\mathrm{SiC} / \mathrm{Si}$
}

\author{
(C) С.А. Кукушкин ${ }^{1-3}$, А.В. Осипов ${ }^{1,3}$, В.Н. Бессолов ${ }^{1,4}$, Е.В. Коненкова ${ }^{1,3,4}$, В.Н. Пантелеев ${ }^{4}$ \\ ${ }^{1}$ Институт проблем машиноведения РАН, \\ Санкт-Петербург, Россия \\ ${ }^{2}$ Санкт-Петербургский государственный политехнический университет, \\ Санкт-Петербург, Россия \\ ${ }^{3}$ Санкт-Петербургский национальный исследовательский университет \\ информационных технологий, механики и оптики, \\ Санкт-Петербург, Россия \\ ${ }^{4}$ Физико-технический институт им. А.Ф. Иоффре РАН, \\ Санкт-Петербург, Россия \\ E-mail: sergey.a.kukushkin@gmail.com \\ (Поступила в Редакцию 11 июля 2016 г.)
}

Обнаружен эффект изменения направления распространения дислокации несоответствия при росте слоев $\mathrm{GaN}$ на поверхности структуры $\mathrm{AlN} / \mathrm{SiC} / \mathrm{Si}(111)$. Эффект заключается в том, что при достижении слоем $\mathrm{GaN}$, растущим на $\mathrm{AlN} / \mathrm{SiC} / \mathrm{Si}(111)$ определенной толщины $\sim 300 \mathrm{~nm}$, дислокации несоответствия первоначально, распространяющиеся вдоль оси роста слоя останавливаются и начинают двигаться в перпендикулярном к оси роста направлению. Построена теоретическая модель зарождения AlN и GaN на грани (111) $\mathrm{SiC} / \mathrm{Si}$, объясняющая эффект изменения направления движения дислокации несоответствия. Обнаружен экспериментально и объяснен теоретически эффект смены механизма зарождения с островкового для AlN на $\mathrm{SiC} / \mathrm{Si}(111)$ на послойный при зарождении слоя $\mathrm{GaN}$ на $\mathrm{AlN} / \mathrm{SiC} / \mathrm{Si}$.

Авторы благодарят за финансовую поддержку Российский научный фонд (грант № 14-12-01102).

Работа выполнена при использовании оборудования Уникальной научной установки (УНУ) „Физика, химия и механика кристаллов и тонких пленок“ ФГУН ИПМаш РАН (г. Санкт-Петербург).

DOI: $10.21883 /$ FTT.2017.04.44266.287

\section{1. Введение}

Хорошо известно $[1,2]$, что карбид кремния является подходящей по многим параметрам и, в частности, по параметрам решетки, подложкой для роста слоев $\mathrm{GaN}$ и AlN. B связи с этим в настоящее время ведутся активные исследования по росту карбида кремния на кремнии [3]. Однако ни один из существующих стандартных методов выращивания $\mathrm{SiC}$ на $\mathrm{Si}$ не дает возможности получать качественные пленки $\mathrm{SiC}$ на кремниевых подложках для создания на их основе приборов силовой электроники или для их использования в качестве буферного слоя при выращивании нитрида галлия и других широкозонных полупроводников [4]. Связано это, как и при росте $\mathrm{GaN}$ на $\mathrm{Si}$ с большой разницей в параметрах решеток подложки кремния и пленки $\mathrm{SiC}$. Эти параметры отличаются более чем на $19 \%$. Более того, в процессе выращивания $\mathrm{SiC}$ этими методами, получается карбид кремния только кубической модификации (3C-SiC), в то время как для роста гексагональных пленок AlN и $\mathrm{GaN}$, желательно иметь на поверхности кремния гексагональный слой $\mathrm{SiC}$, что абсолютно невозможно получить, используя традиционные классические методы роста пленок $\mathrm{SiC}$ на $\mathrm{Si}$.

B paботах $[5,6]$ теоретически предсказан и экспериментально открыт новый метод получения эпитакси- альных пленок нано-SiC на $\mathrm{Si}$, так называемый метод замещения атомов. Данный метод и технология роста нано-SiC, развитая на его основе $[7,8]$, принципиально отличаются от всех существующих на сегодняшний день способов и технологий выращивания монокристаллов, пленок и наноструктур. В основе метода лежит „сборка“ новой матрицы карбида кремния на основе старой матрицы кремния путем частичной замены кремниевых атомов, находящихся в кристаллической матрице подложки на атомы углерода. Для реализации данного подхода в межузельную позицию решетки кремния предварительно внедряют атом углерода, а соседний с ней атомов кремния удаляют, создавая кремниевую вакансию. При этом в приповерхностной области кремниевой решетки образуется ансамбль дилатационных диполей - устойчивых комплексов, состоящих из центров дилатации атома углерода в межузельной позиции и кремниевой вакансии $\left(\mathrm{C}-V_{\mathrm{Si}}\right)$. В кристалле кубической симметрии эти два центра дилатации упруго взаимодействуют друг с другом. В частности, если дилатационные диполи расположены перпендикулярно плоскости (111) кремния, то они притягиваются друг к другу [6,7]. При этом практически вся дилатационная упругая энергия, возникшая из-за внедрения атома углерода и образования вакансии, релаксирует [6,7]. Синтез подобного рода упругих диполей осуществляется при помощи химической реакции 
между кремнием и монооксидом углерода. Скорость данной химической реакции максимальна вдоль направления, вдоль которого дилатационные диполи притягиваются, т. е. вдоль направления [111] подложки Si. После завершения процесса химического превращения механические диполи, выполнившие свою роль, распадаются на пленку карбида кремния и поры под ее поверхностью. Ориентацию пленки при этом задает кристаллическая структура исходной матрицы $\mathrm{Si}$, а не только поверхность подложки, как это обычно реализуется в традиционных методиках выращивания пленок.

\section{2. Структурные и морфологические особенности подложки $\mathrm{SiC} / \mathrm{Si}$}

Отличительной особенностью метода замещения атомов является то, что независимо от первоначальной исходной кристаллографической плоскости $\mathrm{Si}$ на которой синтезировался $\mathrm{SiC}$ обязательно, в качестве одной из плоскостей образуется плоскость [111]. Этот эффект связан с тем, что, упругие диполи атом углеродакремниевая вакансия, всегда расположены вдоль направления [111] перпендикулярно плоскости (111). Именно диполи приводят к резкому ускорению скорости химической реакции образования $\mathrm{SiC}$ вдоль направления [111] кристалла Si.

Важной особенностью метода замещения является наличие пор в $\mathrm{Si}$, которые могут быть заполнены остаточным газом $\mathrm{SiO}$ [7]. Согласно [7] эти поры играют роль демфера при синтезе и охлаждении пленок после их роста. В общем случае поры могут полностью снять упругие термические напряжения. Поэтому подложка нано-SiC/Si, как это описывалось в работах [5-7], становится эластичной. Эластичность подложки нано- $\mathrm{SiC} / \mathrm{Si}$ будет оказывать существенное влияние на зарождение и распространение дислокаций несоответствия в слоях AlN и GaN, выращиваемых на ее поверхности.

В связи с этим целью настоящей работы является постановка специальных экспериментальных исследований по исследованию движения дислокаций несоответствия и построение модели зарождения $\mathrm{AlN}$ и $\mathrm{GaN}$ на грани поверхности (111) буферных слоев нано-SiC, синтезированных топохимическим методом замещения атомов.

\section{3. Методика эксперимента}

Слои нитрида галлия выращивались нами комбинированным методом: на первом этапе методом замещения атомов на подложках $\mathrm{Si}$ ориентаций (111) был выращен слой $\mathrm{SiC}$. Пленки $\mathrm{SiC}$ синтезировались при температуре $1250^{\circ} \mathrm{C}$, время роста составляло $20 \mathrm{~min}$, а сам рост осуществлялся при давлении СО равном $p_{\mathrm{CO}} \geq 1$ Torr [5-9]. Условия роста для пленок на кремнии ориентаций (100) и (111) были одинаковыми. Толщина слоя $\mathrm{SiC}$ составляла $\sim 100 \mathrm{~nm}$. Затем, на выращенных слоях $\mathrm{SiC}$ методом
HVPE [10] был синтезирован буферный слой AlN толщиной $\sim 300 \mathrm{~nm}$ и основной слой $\mathrm{GaN}$ толщиной до $40 \mu \mathrm{m}$. Температура роста буферного слоя AlN была $1080^{\circ} \mathrm{C}$, а температура $\mathrm{GaN}-1050^{\circ} \mathrm{C}$. Эпитаксиальный синтез нитрида алюминия проходил при низких скоростях роста для метода HVPE - 0.5-1 $\mu \mathrm{m} /$ hour при отношении потоков в зоне роста V/III около 500. Рост слоя нитрида галлия проходил при относительно высоких скоростях роста $\sim 40 \mu \mathrm{m} /$ hour и при соотношении $\mathrm{V} / \mathrm{III} \sim 50$.

Слои $\mathrm{GaN}$ исследовались методами рентгеновской дифрактометрии, фотолюминесценции, сканирующей электронной микроскопии (СЭМ) и просвечивающей электронной микроскопии (ПЭМ). Анализ рентгеноструктурных параметров слоя проводился на базе трехкристального рентгеновского спектрометра. ПЭМ-анализ проводился по методике аналогичной [11]. Фотолюминесценция возбуждалась импульсным азотным лазером (длина волны излучения $\lambda=337 \mathrm{~nm}$ ) при $77 \mathrm{~K}$.

\section{4. Структура слоев GaN на AIN/SiC/Si(111)}

На рис. 1 приведено ПЭМ-изображение полярной структуры $\mathrm{GaN}(0001)$, выращенной нами методом HVPE. Исследование полярных $\mathrm{GaN}(0001) / \mathrm{AlN} /$ нано-SiC/Si(111) структур при помощи ПЭМ показало, что эпитаксиальный рост как слоя $\mathrm{AlN}$, так слоя GaN проходит в направлении оси С. В процессе роста дислокации несоответствия прорастают сквозь весь слой $\mathrm{AlN}$ и проникают в слой $\mathrm{GaN}$, но только на глубину $\sim 300 \mathrm{~nm}$. Далее дислокации меняют направление своего движения на направление, перпендикулярное направлению роста пленки, распространяясь практически под прямым углом к оси роста слоя. После поворота даль-

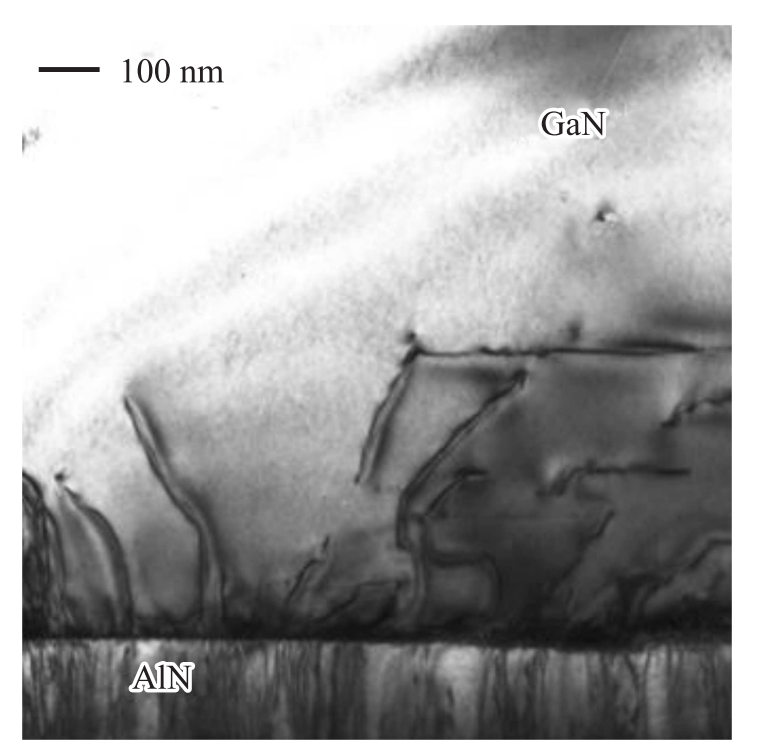

Рис. 1. ПЭМ-изображение слоя $\mathrm{GaN}$, толщиной более $45 \mu \mathrm{m}$ структуры $\mathrm{GaN}(0001) / \mathrm{AlN} /$ нано-SiC/Si(111). 


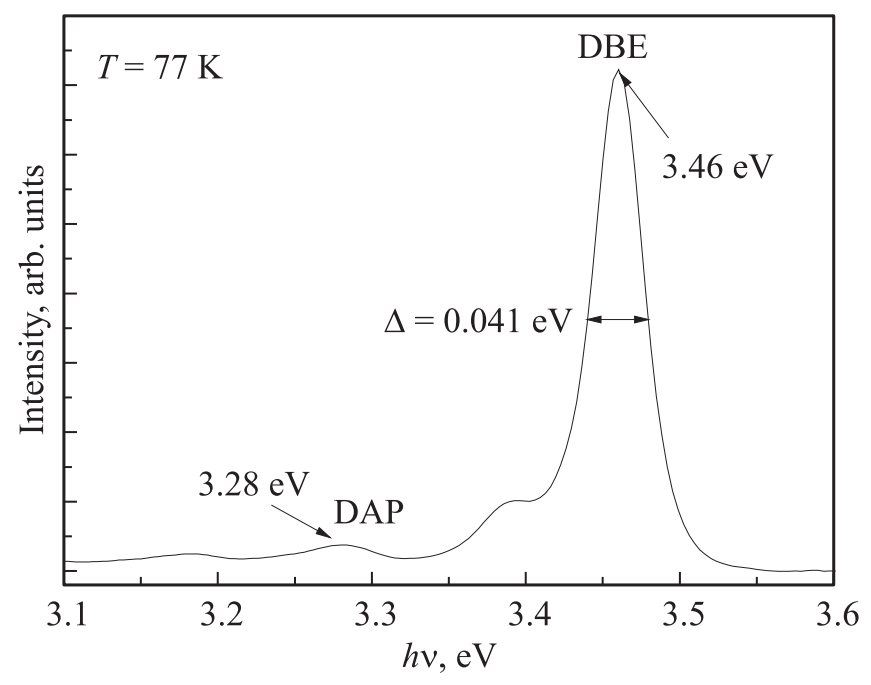

Рис. 2. Спектр фотолюминесценции полярного $\mathrm{GaN}$ при $77 \mathrm{~K}$.

нейший рост дислокаций несоответствия прекращается (рис. 1).

Структуры $\mathrm{GaN}(0001) / \mathrm{AlN} / \mathrm{SiC} / \mathrm{Si}(111)$ по результатам рентгенодифракционного анализа имели полуширину рентгенодифракционных кривых качания для рефлекса (0002) в слоях AlN, равную $\omega_{\theta} \sim 60 \operatorname{arcmin}$, и для слоев $\mathrm{GaN} \sim 500$ arcsec. В спектре фотолюминесценции при $77 \mathrm{~K}$ (рис. 2) имеются линии, соответствующие связанному экситону $\mathrm{DBE}(3.46 \mathrm{eV})$ и донорно-акцепторной рекомбинации $(3.28 \mathrm{eV})$.

По нашему мнению, эффект поворота дислокаций несоответствия (рис. 1) является следствием двух процессов. Подложки нано-SiC/Si, вследствие особой природы метода их синтеза, являются эластичными (податливыми), подстраивающимися под условия выращивания и природу осаждаемых на них пленок. Естественно, что и новая подложка со слоем нано-AlN/нано-SiC/Si будет обладать аналогичными свойствами для дальнейшей гетероэпитаксии слоя $\mathrm{GaN}$. Действительно, при температуре эпитаксии $T=1080^{\circ} \mathrm{C}$ в слое $\mathrm{AlN}$ происходит полная релаксация упругой деформации, возникающей из-за различия постоянных решеток $\mathrm{AlN}$ и $\mathrm{SiC} / \mathrm{Si}(111)$ аналогично, как и при эпитаксии $\mathrm{AlN}$ на $\mathrm{Si}(111)$ [12]. Таким образом, можно предположить, что слой AlN толщиной $300 \mathrm{~nm}$ на $\mathrm{SiC} / \mathrm{Si}(111)$ при температуре эпитаксии становится слабо связанным с теплейтом $\mathrm{SiC} / \mathrm{Si}(111)$ и сам становится эластичным при дальнейшем росте слоя $\mathrm{GaN}$. Рост слоя $\mathrm{GaN}$ на подобного рода подложке до толщины сравнимой с толщиной подложки AIN будет происходить в условиях упругой деформации структуры $\mathrm{AlN} / \mathrm{GaN}$. При толщинах $h_{\mathrm{GaN}}>h_{\mathrm{AlN}}$ произойдет релаксация - переход от упругой деформации к пластической. Этот процесс может привести к резкому изменению роста в тангенциальном направлении и к загибу дислокаций в тангенциальном направлении. В напряженных слоях образуются так называемые фильтрующие свойства по отношению к прорастающим дислокациям.
Идея изгиба прорастающих дислокаций под действием тангенциально направленных сил была реализована в работе [13]. В этой работе внутри слоя GaN была синтезирована напряженная сверхрешетка, которая работала как дислокационный фильтр [13].

\section{5. Модель зарождения и роста пленок AIN и GaN на подложках $\mathrm{SiC} / \mathrm{Si}$}

Из теории роста пленок хорошо известно, что существует три режима их роста: послойный или режим Франка-ван-дер-Мерве, островковый - режим Фольмера-Вебера и промежуточный - режим Странского-Крастанова $[14,15]$. В случае, если атомы осаждаемого вещества связаны между собой сильнее, чем с подложкой, островки сразу зарождаются на поверхности подложки. Этот режим роста называется режимом Фольмера-Вебера. Островковый механизм реализуется, если выполняется условие

$$
\sigma_{\text {sub }}<\sigma_{12}+\sigma_{\text {sub-film }},
$$

где $\sigma_{\mathrm{sub}}-$ свободная энергия единицы поверхности подложки, $\sigma_{12}-$ свободная энергия единицы поверхности конденсированного вещества пленки, $\sigma_{\text {sub-film }}-$ свободная энергия единицы поверхности раздела подложка-пленка.

В цикле работ [16-18] был предложен еще один механизм роста пленок. Согласно теоретическим положениям, развитым в этих работах, оказывается, если трехмерные островки, имеют огранку, то на определенных этапах эволюции островковый механизм роста может смениться послойным. Это означает, что вначале роста пленок, пока зародыши не огранены и имеют незначительный размер, условие (1) выполняется. Затем, по мере роста островков и изменения их морфологии, вызванной образованием огранки на их поверхности, симметрия поверхности островков может измениться. Таким образом, одна из граней островка сама становится подложкой для зарождения на ее поверхности ступени. При росте зародышей по этому механизму верхний слой атомов островков, которые приобрели огранку, начинает играть роль новой подложки. Межфазная энергия при этом обращается в нуль, поскольку ступень образуется на своем собственном материале. В общем случае, если ступень образуется на чужеродном материале или на грани другой ориентации, то необходимо добавить межфазную энергию между ступенью и чужеродной гранью. Согласно [16-18] это может привести к смене знака в неравенстве (1) на противоположный, т. е.

$$
\sigma_{\text {sub-is }}>\sigma_{\text {st }}+\sigma_{\text {st-sub-is }},
$$

где $\sigma_{\mathrm{st}}-$ поверхностная энергия ступени, $\sigma_{\mathrm{sub}-\text { is }}$ в данном случае, обозначает поверхностную энергию какой-либо из граней островка с собственным паром, $\sigma_{\mathrm{st}-\mathrm{sub}-\mathrm{is}}-$ поверхностная энергия между ступенью и гранью островка. 

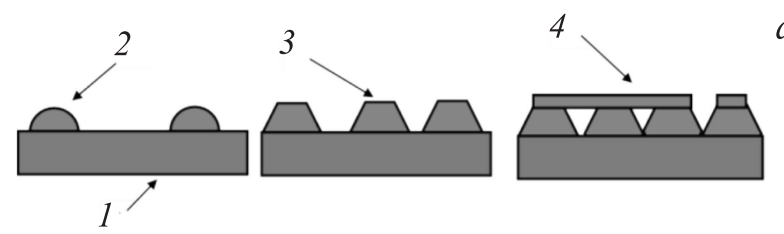

$a$

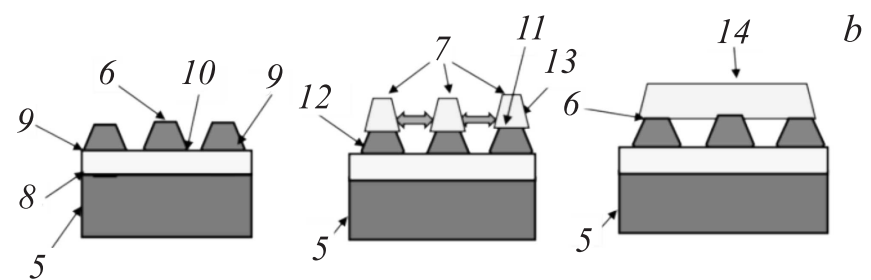

Рис. 3. (a) режим роста пленок, при котором происходит переход от островкового роста к послойному. 1 - подложка, 2 - куполообразные островки, 3 - ограненные островки, 4 - послойный рост [21-23]. (b) последовательные стадии роста слоя $\mathrm{GaN}$ по механизму, предложенному в работах [21-23], 5 - подложка $\mathrm{Si} / \mathrm{SiC}, 6$ - ограненные островки $\mathrm{AlN}$, плоскость (0001) AlN, 7 - ограненные островки AlN с зародышевым слоем $\mathrm{GaN}$, плоскость (0001) $\mathrm{GaN}, 8-$ плоскость (111) Si, 9 - плоскость (111) $\mathrm{SiC}, 10$ - плоскость (1000) AlN, 11 - плоскость (1000) GaN, 12 - плоскость (112̄0) AlN, 13 - плоскость (112̄0) GaN, 14 - послойный рост пленки $\mathrm{GaN}$; стрелки указывают направление роста слоя $\mathrm{GaN}$ и геометрическое место точек, где возникает дислокация несоответствия между $\mathrm{AlN}$ и $\mathrm{GaN}$; из этой дислокации и образуется двумерный зародыш ступени $\mathrm{GaN}$, на основе которой и начинается $2 D$ рост слоя $\mathrm{GaN}$.

Это механизм роста схематично изображен на рис. 3, $a$, а на рис. $3, b$ модель роста слоев $\mathrm{AlN}$ и $\mathrm{GaN}$ на подложке $\mathrm{Si} / \mathrm{SiC}$ в соответствии с теоретическими положениями [16-18].

Нам удалось экспериментально реализовать данный механизм роста $\mathrm{GaN}(0001) / \mathrm{AlN} / \mathrm{SiC} / \mathrm{Si}(111)$, что хорошо видно из электронной микрофотографии высокого разрешения слоя $\mathrm{GaN}(0001) / \mathrm{AlN} / \mathrm{SiC} / \mathrm{Si}(111)$, приведенной в работе [19]. В работе [19] было показано, что пирамидальные зародыши нитрида алюминия на слое $\mathrm{SiC}$ расположены отдельно друг от друга и покрыты сверху толстым слоем GaN. Анализ микроэлектронограммы в [19] показал, что слои являются эпитаксиальными и в гетероструктуре в области интерфейса в выполняются следующие эпитаксиальные соотношения $\mathrm{Si}(111)\|3 \mathrm{C}-\mathrm{SiC}(111)\| \operatorname{AlN}(0001) \| \mathrm{GaN}(0001)$ и

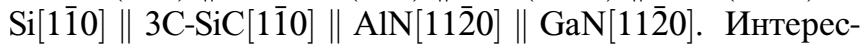
но отметить, что точно такие же соотношения, выполняются и при $2 D$ росте $\mathrm{GaN}$ на подложке сапфира со слоем AlN, в то время как при росте GaN на сапфире без буферного слоя AlN растут трехмерные островки $\mathrm{GaN}$ с иным ориентационным соотношением [20].

Для реализации данного механизма, согласно расчетам, проведенным в [16-18] необходимо, чтобы в процессе зарождения $\mathrm{AlN}$ на подложке $\mathrm{SiC} / \mathrm{Si}$ выполнялось условие (1). При росте $\mathrm{AlN}$ на подложке
$\mathrm{SiC} / \mathrm{Si} \sigma_{1 \mathrm{st}}=\sigma_{\mathrm{SiC}} ; \sigma_{12}=\sigma_{\mathrm{AlN}} ; \sigma_{2 \mathrm{st}}=\sigma_{\mathrm{SiC}-\mathrm{AlN}}$. Согласно работам [21,22] поверхностная энергия грани (0001) $\mathrm{AlN} \sigma_{\mathrm{AIN}}=4.51 \mathrm{Jm}^{-2}$. Поверхностная энергия грани $\mathrm{SiC}(111)$ точно неизвестна. Расчет, выполненный в работе [23] дал следующее значение $\sigma_{\mathrm{SiC}(111)}^{s}=2.58 \mathrm{Jm}^{-2}$. Это значение близко к экспериментальному значению $\sigma_{\mathrm{SiC}(111)}^{S}$, приведенному в обзоре [24]. Из экспериментальных данных, полученных нами ранее [19] (см. рис. 3 и рис. 7 в статье [19]) следует, что форма зародышей AlN близка по форме к пирамиде. Если аппроксимировать эту форму зародыша полусферой с углом смачивания, близким к $45^{\circ}$, то из условия $\cos \theta=\left(\sigma_{1 \mathrm{st}}-\sigma_{2 \mathrm{st}}\right) / \sigma_{12}$ легко получить, что в этом случае $\sigma_{2 \mathrm{st}}=\sigma_{\mathrm{SiC}-\mathrm{AlN}}$ действительно приблизительно равна $\sigma_{2 \mathrm{st}}=\sigma_{\mathrm{SiC}-\mathrm{AlN}} \approx 1.7 \mathrm{Jm}^{-2}$. Будем использовать это значение межфазной энергии. При этих значениях поверхностной энергии условие (1) заведомо выполняется. Поверхностная энергия существенно зависит и от условий контакта поверхности кристалла с различными газами, в частности контакта с водородом. Синтез AIN мы проводили с предварительной обработкой $\mathrm{SiC}$ в водородной среде, поэтому поверхностная энергия подложки должна быть еще меньше. Таким образом, на поверхности нано-SiC/Si(111) растет зародышевый, островковый слой AlN. Ситуация кардинальным образом изменяется при последующем наращивании слоя GaN на поверхность зародышей AlN. Поверхностная энергия грани (0001) GaN [21-23,25] лежит в области между 4-5 $\mathrm{Jm}^{-2}$. Поверхностная энергия грани $(11 \overline{2} 0) \sigma_{\mathrm{GaN}}=3.23 \mathrm{Jm}^{-2}[22]$, а для соответсвующей ей грани (112̄0) AlN $\sigma_{\mathrm{AlN}}=3.1 \mathrm{Jm}^{-2}$ [22]. Вицинальные грани, и в частности грань $(11 \overline{2} 0)$, покрываются ступенями, ориентированными по направлению [0001]. Если на грани (0001) AlN с $\sigma_{\mathrm{AIN}}=4.51 \mathrm{Jm}^{-2}$ образуется ступень $\mathrm{GaN}$ с направлением [0110], поверхностная энергия которой [22] $\mathrm{GaN} \sigma_{\mathrm{GaN}}=3.11 \mathrm{Jm}^{-2}$, то вследствие близости параметров решеток этих веществ и их химических свойств можно предположить, что условие (2) для этой системы будет выполняться. Действительно, в этом случае можно полагать, что $\sigma_{\mathrm{st}-\mathrm{sub}-\mathrm{is}}<1 \mathrm{Jm}^{-2}$. Это означает, что островок AlN будет являться подложкой для послойного роста $\mathrm{GaN}$. C другой стороны для грани (112̄0) это условие не выполнятеся, поскольку она покрыта ступенями орентации [0001], поверхностная энергия которых значительно выше. Это означает, что на данной поверхности, будет происходить островковый механизм зарождения. На границе пересечения этих граней с различным механизмом роста будет образовываться ростовой дефект (дислокация, поверхностная вакансия). Сам же зародыш $\mathrm{GaN}$ будет иметь форму приплюснутой сверху „шапочки“, надетой на верхушку приамидального зародыша AlN, так как это изображено на рис. 3. Эта „шапочка“ будет охватывать вершину пирамиды. На месте дефекта роста образуется ступень ориентации [0110], которая будет в дальнейшем расти в плоскости (0001). Поскольку поверхностная энергия этой ступени ниже, чем ступеней других ориентаций 
(здесь имеется в виду из ступеней, образующихся в данном ростовом процессе) [14], то и ее скорость роста будет выше. Ступени соседних „шапочек“ со временем будут сливаться друг с другом, образуя фигуру, обозначенную номером 14 на рис. $3, b$. Для реализации данного механизма роста согласно [16-18] необходимо, чтобы на поверхности AlN выполнялось следующее условие для линейного размера грани $x$ :

$$
x>4 r_{\mathrm{cr}},
$$

где

$$
r_{\mathrm{cr}}(R)=\frac{\sigma_{\mathrm{st}} \Omega_{s}}{\Delta \mu(R)}
$$

- критический размер двумерного зародыша GaN, зарождающегося на кристаллической грани зародыша AlN радиуса $R$ (в нашем случае это средний размер грани) в месте выхода на поверхность грани винтовой дислокации, $\Delta \mu(R)$ - разность химических потенциалов газовой и кристаллической фазы $\mathrm{GaN}$ радиуса $R$, рассчитанная на один атом, $\sigma_{\mathrm{st}}$ - поверхностная энергия ступени двумерного зародыша $\mathrm{AlN}$; $\Omega_{s}$ - объем на атом в островке. Условие (3) означает, что образующийся на поверхности грани AlN двумерный зародыш $\mathrm{GaN}$ должен иметь более чем в 4 раза меньший критический размер, чем линейный размер грани $\mathrm{AlN}$, на которой он растет. В противном случае данный механизм не реализуется. Для строгого описания данного процесса можно воспользоваться теорией зарождения кристаллов в условиях ограниченных объемов, развитой Дубровским. Эта теория изложена им в монографии [25]. Здесь же проведем некоторые оценки, используя теорию многокомпонентного зарождения [5].

При росте AlN методом хлоридной эпитаксии пересыщение $\xi_{\text {AIN }}$ имеет вид

$$
\xi_{\mathrm{AIN}}=k T \ln \frac{P_{\mathrm{NH}_{3}} P_{\mathrm{AlCl}_{3} K_{P}^{\mathrm{AlN}}}}{P_{\mathrm{HCl}}^{3}} .
$$

Аналогичное выражение для $\mathrm{GaN}$ будет выглядеть таким образом

$$
\xi_{\mathrm{GaN}}=k T \ln \frac{P_{\mathrm{GaCl}} P_{\mathrm{NH}_{3}} K_{P}^{\mathrm{GaN}}}{P_{\mathrm{HCl}} P_{\mathrm{H}_{2}}}
$$

где

$$
K_{P}^{\mathrm{AlN}}=\frac{\left(P_{\mathrm{HCl}}^{0}\right)^{3}}{P_{\mathrm{NH}_{3}}^{0} P_{\mathrm{AlCl}_{3}}^{0}}
$$

- константа равновесия образования AlN по химической реакции $\mathrm{AlCl}_{3}+\mathrm{NH}_{3}=\mathrm{AlN}^{(s)}+3 \mathrm{HCl}$, a

$$
K_{P}^{\mathrm{GaN}}=\frac{P_{\mathrm{H}_{2}}^{0} P_{\mathrm{HCl}}^{0}}{P_{\mathrm{GaCl}}^{0} P_{\mathrm{NH}_{3}}^{0}}
$$

- константа равновесия образования $\mathrm{GaN}$ по химической реакции $\mathrm{GaCl}+\mathrm{NH}_{3}=\mathrm{GaN}^{(s)}+\mathrm{HCl}+\mathrm{H}_{2}$. Здесь $-P_{\mathrm{H}_{2}}^{0}, P_{\mathrm{HCl}}^{0}, P_{\mathrm{GaCl}}^{0}, P_{\mathrm{AlCl}_{3}}^{0}, P_{\mathrm{NH}_{3}}^{0}-$ равновесные при температуре синтеза давления водорода $\left(\mathrm{H}_{2}\right)$, хлористого водорода $(\mathrm{HCl})$, монохлорида галлия $(\mathrm{GaCl})$, хлорида алюминия $\left(\mathrm{AlCl}_{3}\right)$ и аммиака $\left(\mathrm{NH}_{3}\right)$ соответственно; $P_{\mathrm{H}_{2}}, P_{\mathrm{HCl}}, P_{\mathrm{GaCl}}, P_{\mathrm{AlCl}_{3}}, P_{\mathrm{NH}_{3}}-$ рабочие давления $\mathrm{H}_{2}$, $\mathrm{HCl}, \mathrm{GaCl}, \mathrm{AlCl}_{3}, \mathrm{NH}_{3}$ соответственно. Оценим значения величин $\xi_{\mathrm{AIN}}$ и $\xi_{\mathrm{GaN}}$. Согласно расчетам, выполненным в работе [26], $K_{P}^{\mathrm{GaN}} \approx 10$. Значение константы $K_{P}^{\mathrm{AIN}}$ можно получить на основании значений равновесных давлений газов. Равновесные давления $P_{\mathrm{H}_{2}}^{0}, P_{\mathrm{HCl}}^{0}, P_{\mathrm{GaCl}}^{0}, P_{\mathrm{AlCl}_{3}}^{0}$, $P_{\mathrm{NH}_{3}}^{0}$ были рассчитаны в работе [27] для AlN и в работе [28] для $\mathrm{GaN}$ для широкого диапазона температур и при различных отношения компонентов V/III в зоне роста. Мы синтезировали слои AlN при отношении потоков компонентов V/III в зоне роста, равном $\mathrm{V} / \mathrm{III} \approx 500$. Температура синтеза AIN была $T=1080^{\circ} \mathrm{C}$. Константа химической реакции при неизменной температуре и неизменном общем давлении, как известно, является величиной постоянной. Поэтому, если известны равновесные давления $P_{\mathrm{H}_{2}}^{0}, P_{\mathrm{HCl}}^{0}, P_{\mathrm{NH}_{3}}^{0}, P_{\mathrm{GaCl}}^{0}$, $P_{\mathrm{AlCl}_{3}}^{0}$ при любом соотношении компонентов V/III можно вычислить константу равновесия. При $T \approx 1100^{\circ} \mathrm{C}$, достаточно близкой к экспериментальному значению температуры и отношении $\mathrm{V} / \mathrm{III}=1$, согласно [26], $P_{\mathrm{H}_{2}}^{0} \approx 1 \mathrm{~atm}, \quad P_{\mathrm{NH}_{3}}^{0} \approx 5 \cdot 10^{-5} \mathrm{~atm}, \quad P_{\mathrm{HCl}}^{0} \approx 8 \cdot 10^{-3} \mathrm{~atm}$, $P_{\mathrm{AlCl}_{3}}^{0} \approx 5 \cdot 10^{-5} \mathrm{~atm}$. Отсюда для константы равновесия имеем $K_{P}^{\mathrm{AlN}} \approx 3 \cdot 10^{2} \mathrm{~atm}$. Мы выращивали пленки $\mathrm{AlN}$ и $\mathrm{GaN}$ при скорости потока $\mathrm{H}_{2}$, равной $1.7 \mathrm{slm}$; скорости потока $\mathrm{NH}_{3}-1.2 \mathrm{slm}$; скорости потока $\mathrm{HCl}$ над слоем (алюминия и галлия) $-10^{-2} \mathrm{slm}$. Считая среду, в которой происходит рост пленок $\mathrm{AlN}$ и $\mathrm{GaN}$, идеальным газом, можно воспользоваться уравнением состояния идеальных газов, записанным через объемную его скорость поступления [29] $P V=\left(\Sigma_{i} n_{i}\right) R T$, где $V$ - обозначает объемную скорость подачи газов в $\operatorname{Lsec}^{-1}$, давление $P$ измеряется в атмосферах, а $n_{i}$ - объем молей, вводимых в реактор в единицу времени. Это позволяет вычислить рабочие давления газов. Можно показать, что при скорости потока $\mathrm{HCl}$ в $10^{-2} \mathrm{slm}$ давление поступающего газообразного $\mathrm{HCl}$ равно $P_{\mathrm{HCl}} \approx 6 \cdot 10^{-3} \mathrm{~atm}$. Соответственно давление $P_{\mathrm{AlCl}_{3}}$ в потоке составляет $P_{\mathrm{AlCl}_{3}} \approx 2 \cdot 10^{-3} \mathrm{~atm}$, что следует из расчета доли образующегося $\mathrm{AlCl}_{3}$, выполненного в работе [26]. Давление $P_{\mathrm{NH}_{3}} \approx 7 \cdot 10^{-1} \mathrm{~atm}$, а давление $P_{\mathrm{HCl}}$ положим равным входящему давлению, которое близко к равновесному при V/III $\sim 500$ и $T \approx 1100^{\circ} \mathrm{C}$, т. е. $P_{\mathrm{HCl}} \approx 6 \cdot 10^{-3} \mathrm{~atm}$. Отсюда и уравнения (5) следует, что величина

$$
\ln \frac{P_{\mathrm{NH}_{3}} P_{\mathrm{AlCl}_{3}} K_{P}^{\mathrm{AlN}}}{P_{\mathrm{HCl}}^{3}},
$$

входящая в химическое сродство (5) принимает следующее значение

$$
\ln \frac{P_{\mathrm{NH}_{3}} P_{\mathrm{AlCl}_{3}} K_{P}^{\mathrm{AlN}}}{P_{\mathrm{HCl}}^{3}} \approx 14,
$$

а химическое сродство при температуре синтеза $\xi_{\mathrm{AIN}} \approx 2.5 \cdot 10^{-19} \mathrm{~J}$. 
Слои $\mathrm{GaN}$ выращивались нами при отношении потоков компонентов V/III в зоне роста V/III $\sim 50$. При этом отношении компонентов, согласно данным работы [28] и анализу, проведенному выше, для AlN получаем величину

$$
\ln \frac{P_{\mathrm{GaCl}} P_{\mathrm{NH}_{3}} K_{P}^{\mathrm{GaN}}}{P_{\mathrm{HCl}} P_{\mathrm{H}_{2}}} \approx 40 .
$$

Соответствующее этому значению химическое сродство при температуре синтеза равно $\xi_{\mathrm{GaN}} \approx 7 \cdot 10^{-19} \mathrm{~J}$.

Скорость образования (скорость нуклеации) зародышей $\mathrm{AlN}$ и $\mathrm{GaN}$ определяется Больцмановским распределением $I \sim \exp \left(-\Delta F^{*} / k T\right)$, где $\Delta F^{*}-$ свободная энерия образования зародыша критического размера. При зарождении $\mathrm{AlN}$ на поверхности нано-SiC возможны следующие две ситуации.

1. Образуется двумерный дискообразный островок высота которого, равна высоте моноатомной ступени. Мы положили для вюрцитных кристаллов, что высота ступени равна параметру решетки $a_{c}$ вдоль оси с; у AlN $a_{c}=4.98 \cdot 10^{-10} \AA$, a y GaN $a_{c}=5.18 \cdot 10^{-10} \AA$.

2. Образуется трехмерный островок в форме полусферы, которая вследствие большого поверхностного натяжения $\mathrm{AlN}$ или $\mathrm{GaN}$ покрывается кристаллическими гранями и приобретает форму пирамиды (см. рис. 3). Свободная энергия образования двумерного островка согласно $[25,29,30]$ имеет вид

$$
\Delta F_{2 D}^{*}=\frac{\pi\left(a_{c} \sigma_{12}\right)^{2}}{\frac{\xi_{\mathrm{AlN}, \mathrm{GaN}}}{\Omega} a_{c}-\left(\sigma_{12}+\sigma_{2 \mathrm{st}}-\sigma_{1 \mathrm{st}}\right)},
$$

в котором $\Omega$ - объем на атом в AlN или GaN. Если на подложке образуется трехмерный островок в форме полусферы, то свободная энергия будет иметь вид

$$
\Delta F_{3 D}^{*}=\frac{16 \pi \sigma_{12}^{3}}{3\left(\frac{\xi_{\mathrm{AlN}, \mathrm{GaN}}}{\Omega}\right)^{2}} \Phi(\theta)
$$

где $\Phi(\theta)$ - функция контактного угла $\theta, \Phi(\theta)=$ $=\left(2-3 \cos \theta+\cos ^{3}(\theta)\right) / 4 ; \cos \theta=\left(\sigma_{1 \mathrm{st}}-\sigma_{2 \mathrm{st}}\right) / \sigma_{12}$.

Сделаем простейшие оценки величин $\Delta F_{2 D}^{*}$ и $\Delta F_{3 D}^{*}$ для зарождения $\mathrm{AlN}$ на нано-SiC/Si. При выводе выражений $\Delta F_{2 D}^{*}$ и $\Delta F_{3 D}^{*}$ мы не учитывали упругую энергию, возникающую из-за различия в параметрах решеток пленки и подложки. Деформация $\varepsilon$, вызванная различием в параметрах решеток (0001) AlN и (111) кубического $\mathrm{SiC}$ или (0001) гексагонального $\mathrm{SiC}$ составляет $1 \%$. Деформация решеток (0001) AlN и (0001) GaN не превышает $2 \%$. Это весьма малые значения деформации, которые не будут существенным образом оказывать влияние на процесс зарождения островков $\mathrm{AlN}$ и GaN. Для других подложек, например для подложки $\mathrm{Si}$ (111) и пленки AlN (0001), деформация составляет 19\%. Это большая величина и ее необходимо учитывать при вычислении свободной энергий зарождения новой фазы. В самом простом случае упругую составляющую энергии зародыша, имеющего форму диска высотой в один монослой $a_{c}$, можно оценить так, как это было вычислено в работе [7]. С учетом упругой составляющей выражение (7) можно переписать в виде

$$
\Delta F_{2 D}^{*}=\frac{\pi\left(a_{c} \sigma_{12}\right)^{2}}{\left(\frac{\xi_{\mathrm{AIN}, \mathrm{GaN}}}{\Omega}-\frac{\mu^{\mathrm{st}} \varepsilon^{2}}{2}\right) a_{c}-\left(\sigma_{12}+\sigma_{2 \mathrm{st}}-\sigma_{1 \mathrm{st}}\right)}
$$

где $\mu^{\text {st }}-$ модуль сдвига подложки; у кремния $\mu^{\mathrm{Si}}=0.52 \cdot 10^{11} \mathrm{~N} / \mathrm{m}^{2}$. Аналогичным образом можно обобщить и выражение (8). Отметим, что член с упругой энергией был нами вычислен приближенно, поэтому он пригоден только для оценок. Как будет видно из дальнейшего, это выражение в целом качественно верно отражает природу процесса зарождения новой фазы на различных подложках. Пленка нано-SiC/Si, как уже отмечалось, лишь частично сопряжена с поверхность подложки $\mathrm{Si}$. Мы поэтому имеем основание полагать, что для данной системы подложка $\mathrm{Si}$, во всяком случае на раннем этапе образования зародышей, не играет особой роли в формировании упругой энергии. В связи с этим положим, что модуль $\mu^{\mathrm{st}}$, зависит только от механических модулей слоя $\mathrm{SiC}$, т.е. $\mu^{\mathrm{st}}=\mu^{\mathrm{SiC}}=1.8 \cdot 10^{11} \mathrm{Nm}^{-2}$ [7]. Однако в этом случае формулу (9) можно использовать только если зародыши AlN имеют размер меньший или сравнимый с толщиной слоя $\mathrm{SiC}$. В нашем случае, как правило, толщина слоя $\mathrm{SiC}$ не превышает $100 \mathrm{~nm}$.

Величина $\left(\sigma_{12}+\sigma_{2 \mathrm{st}}-\sigma_{1 \mathrm{st}}\right)$, входящая в уравнение $(7)$, c учетом приведенных выше значений поверхностных энергий, равна $\left(\sigma_{12}+\sigma_{2 \mathrm{st}}-\sigma_{1 \mathrm{st}}\right)=\left(\sigma_{\mathrm{AlN}}+\sigma_{\mathrm{SiC}-\mathrm{AlN}}\right.$ $\left.-\sigma_{\mathrm{SiC}(111)}^{s}\right) \sim 1.3 \mathrm{Jm}^{-2}$. Отсюда следует, что, во-первых, условие (1) удовлетворяется, что указывает на то, что рост AlN происходит по механизму Фольмера-Вебера. При этом $\cos \theta=0.7$, а $\theta \approx 45^{\circ}$. Для этого угла коэффициент формы $\Phi(\theta)=0.05$. Поскольку островки AlN образуются в виде пирамидок, покрытых гранями (11) 0$)$, поверхностная энергия которых порядка $\sigma_{\mathrm{AIN}(11 \overline{2} 0)}=3 \mathrm{Jm}^{-2}[21]$, примем, что величина $\sigma_{12}$, входящая в выражение $(8)$, равна $\sigma_{12}=\sigma_{\mathrm{AlN}(11 \overline{2} 0)}$. У AlN объем на атом составляет величину $\Omega \approx 2 \cdot 10^{-29} \mathrm{~m}^{3}$. Отсюда следует, что $\Delta F_{3 D}^{*} \approx 5 \cdot 10^{-19} \mathrm{~J}$. Свободная энергия зарождения $2 D$ островка $\mathrm{AlN} \Delta F_{2 D}^{*}$ при этих значениях поверхностной энергии равна $\Delta F_{2 D}^{*} \approx 3 \cdot 10^{-18} \mathrm{~J}$. Таким образом, свободная энергия $\Delta F_{2 D}^{*}$ почти на порядок больше свободной энергии $\Delta F_{3 D}^{*}$. Следовательно, при данных условиях синтеза в основном будут зарождаться островки AlN в форме пирамиды.

Рассмотрим теперь зарождение GaN. При зарождении $\mathrm{GaN}$ на поверхности зародышей $\mathrm{AlN}$, слой $\mathrm{AlN}$, как показано выше, сам является подложкой. Для послойного роста слоя $\mathrm{GaN}$ необходимо одновременное выполнение условий (3) и (4). Это означает, что для реализации данного механизма роста образующийся на поверхности грани $\mathrm{AlN}$ двумерный зародыш $\mathrm{GaN}$ должен иметь в 4 раза меньший критический размер, чем линейный размер грани AlN, на которой он растет. В нашем случае это условие выполняется, поскольку критический размер зародыша $\mathrm{AlN}$, соответствующий энергии (8) 
$r_{\text {cr AIN }} \approx 5 \mathrm{~nm}$, а критический размер двумерного зародыша $\mathrm{GaN}(7) r_{\mathrm{crGaN}} \approx 1 \mathrm{~nm}$. Таким образом, имеется возможность для двумерного зарождения $\mathrm{GaN}$ на грани AlN. Отметим, что после зарождения островков AlN они еще достаточно долго растут, увеличиваясь в размерах. В конце процесса синтеза, согласно экспериментальным данным [19], размер островков AlN становится равным примерно $r_{\text {cr AlN }} \approx 40-50 \mathrm{~nm}$. После образования одного-двух монослоев $\mathrm{GaN}$ на поверхности зародышей $\mathrm{AlN}$, дальнейший рост слоя $\mathrm{GaN}$ будет происходить уже на поверхности своего „родного“ материала. На начальной стадии зарождения $\mathrm{GaN}$ образуется дислокация, которая и порождает двумерный зародыш $\mathrm{GaN}$ или ступень роста (7) [16-18]. Причины, вызвавшие возникновение дислокации несоответствия в начале зарождения, а именно различие в параметрах решеток AlN и $\mathrm{GaN}$, будут с увеличением толщины слоя $\mathrm{GaN}$ затухать. Это связано с тем, что пленка GaN зарождается на набольших участках граней $\mathrm{AlN}$ и с увеличением толщины слоя слой „забывает“ исходную затравку (зародыш AlN) и растет практически в свободных от деформаций условиях. Слой $\mathrm{GaN}$ подобен мосту на сваях, практически не напряжен. Этот механизм релаксации упругих напряжений подобен механизму релаксации напряжений в пленке $\mathrm{SiC}$ на $\mathrm{Si}$, выращенной методом замещения атомов [5-9]. Для удовлетворения условию (3) необходимо вести синтез $\mathrm{GaN}$ в условиях высокого пересыщения при большой скорости роста, что и было реализовано нами в настоящей работе. Реализация подобного механизма позволяет выращивать слои $\mathrm{GaN}$ толщиной $40 \mu \mathrm{m}$ и более, практически не содержащие дислокаций, перпендикулярных оси роста с. Если оценить величину упругой энергии $\mu^{\mathrm{st}} \varepsilon^{2} / 2$, возникающей при зарождении как слоя AlN, так и слоя $\mathrm{GaN}$, то она будет составлять примерно одну десятитысячную от величины первого члена в знаменателе (7). Если же оценить аналогичную энергию для чистой кремниевой подложки, то упругая энергия будет лишь незначительно меньше, чем первый член в знаменателе (7), а именно $\mu^{\text {st }} \varepsilon^{2} / 2 \approx 7 \cdot 10^{9} \mathrm{~J}$, в то время как первый член $\xi_{\mathrm{AlN}} / \Omega \approx 1 \cdot 10^{10} \mathrm{~J}$. С учетом того, что поверхностная энергия грани (111) $\mathrm{Si}$ $\sigma_{\mathrm{Si}(111)} \approx 2 \mathrm{Jm}^{-2}$ [7] знаменатель в формуле (7) будет отрицательным. Это означает, что на $\mathrm{Si}$ зарождение $2 D$ островков AlN при данных условиях роста методом HVPE мало вероятно. Необходимо либо специальным образом модифицировать поверхность $\mathrm{Si}$, либо еще увеличивать пересыщение. В работе [30] было показано (для однокомпонентных систем), что если величина относительного пересыщения превышает единицу, то система „заходит“ в область сильной метастабильности. При этом возможен спинодальный распад и рассмотрение зарождения на основе классической теории (5)-(9) не совсем корректно. В случае многокомпонентных систем с химическими реакциями, очевидно, процесс протекает более сложно, что требует дополнительного анализа. Вполне возможно, что образование и $\mathrm{AlN}$, и $\mathrm{GaN}$ будет протекать при больших пересыщениях через промежуточные метастабильные вещества. При синтезе AlN такой промежуточной фазой может быть, например, $\mathrm{NH}_{4} \mathrm{AlCl}_{4}$. Следует отметить, что реакция образования $\mathrm{GaN}$, приведенная выше, с химической точки зрения обязательно должна протекать через ряд параллельных и последовательных стадий, в результате которых может образовываться ряд плотных (жидких или твердых) фаз, исследованию которых мы посвятим отдельное сообщение. Эти плотные фазы будут снижать поверхностную энергию между ними и зародышем. При этом пересыщение должно вычисляться исходя из концентрации молекул данных фаз. Кроме того, необходимо определить промежуточные химические реакции так, как это было сделано для роста пленки $\mathrm{SiC}$ на $\mathrm{Si}$ методом замещения атомов [9]. Однако в соответствии с законом действующих масс Гульдберга-Вааге для качественных оценок мы можем использовать только начальные и конечные состояния химической реакции, чем мы и воспользовались в настоящей работе.

\section{6. Заключение}

Итак, вследствие особой физической природы слоя нано-SiC и способа его синтеза, подложка, т.е. нано$\mathrm{SiC} / \mathrm{Si}(111)$, обладает рядом особых свойств, отличающих ее от других подложек. Этими свойствами являются следующие.

1. Подложка, подстраиваться под условия выращивания пленок $\mathrm{AlN}$ и $\mathrm{GaN}$, что приводит к резкому снижению плотности дислокаций несоответствия в слое $\mathrm{GaN}$ и позволяет при высоких пресыщениях сменить механизм роста с островкового на послойный, или, более точно, на overgrowth.

2. Подложка нано-SiC/Si с буферным слоем AlN является гетероструктурой для дальнейшего выращивания на ее поверхности ненапряженных слоев GaN. Это означает, что случае сама структура $\mathrm{AlN} / \mathrm{SiC} / \mathrm{Si}(111)$ является фундаментом для дальнейшего роста низкодефектных пленок GaN. Этот фундамент, состоящий из зародышевого слоя $\mathrm{AlN}$, лежащего на слое нано-SiC, в свою очередь лежащего на пористом $\mathrm{Si}$, напоминает фундамент дома, построенного на зыбкой почве или вечной мерзлоте. Этот фундамент подстраивается под внешние условия, сохраняя свою структуру упорядоченной и оставаясь при этом ненапряженным.

Авторы благодарят Л.М. Сорокина, А.Е. Калмыкова и А.В. Мясоедова за проведенные ПЭМ-измерения.

\section{Список литературы}

[1] S. Pimputkar, J.S. Speck, S.P. DenBars, S. Nakamura. Nature Photon 3, 180 (2009).

[2] J. Komiyama, D. Yoshihisa, S. Suzuki, K. Toru, H. Nakanishi. Appl. Phys. Lett. 88, 091901 (2006).

[3] G. Ferro. Solid State Mater. Sci. 40, 56 (2015).

[4] A. Severinoa, C. Lockeb, R. Anzalonea, M. Camardaa, N. Pilusoa, A.La Magnaa, S.E. Saddowb, G. Abbondanzac, G.D'Arrigoa, F.La Viaa. ECS Transactions 35, 99 (2011). 
[5] С.А. Кукушкин, А.В. Осипов. ФТТ 50, 1188 (2008).

[6] S.A. Kukushkin, A.V. Osipov. J. Appl. Phys. 113, 024909 (2013).

[7] S.A. Kukushkin, A.V. Osipov. J. Phys. D 47, 313001 (2014).

[8] С.А. Кукушкин, А.В. Осипов, Н.А. Феоктистов. ФТТ 56, 1457 (2014).

[9] С.А. Кукушкин, А.В. Осипов. ФТТ 58, 725 (2016).

[10] V.N. Bessolov, E.V. Konenkova, S.A. Kukushkin, A.V. Osipov, S.N. Rodin. Rev. Adv. Mater. Sci. 38, 75 (2014).

[11] L.M. Sorokin, A.V. Myasoedov, A.E. Kalmykov, D.A. Kirilenko, V.N. Bessolov, S.A. Kukushkin. Semicond. Sci. Technol. 30, 114002 (2015).

[12] В.Н. Бессолов, В.Ю. Давыдов, Ю.В. Жиляев, Е.В. Коненкова, Г.Н. Мосина, С.Д. Раевский, С.Н. Родин, Ш. Шарофидинов, М.П. Щеглов, Р.H. Seok, К. Masayoshi. ПЖТФ 31, 30 (2005).

[13] J.W. Matthews, A.E. Blackeslee, S. Mader. Thin Solid Films 33, 253 (1976).

[14] А.А. Чернов, Е.И. Гиваргизов, Х.С. Багдасаров. Образование кристаллов. Современная кристаллография. Т. 3 / Под ред. В.К. Вайнштейна, А.А. Чернова, Л.А. Шувалова. Наука, М. (1980). 408 c.

[15] S.A. Kukushkin, A.V. Osipov. Progr. Surf. Sci. 151, 1 (1996).

[16] S.A. Kukushkin, T.V. Sakalo. Acta Metal. Mater. 41, 1237 (1993).

[17] S.A. Kukushkin, T.V. Sakalo. Acta Metal. Mater. 42, 2797 (1994).

[18] T.V. Sakalo, S.A. Kukushkin. Appl. Surf. Sci. 92, 350 (1996).

[19] Л.М. Сорокин, А.Е. Калмыков, В.Н. Бессолов, Н.А. Феоктистов, А.В. Осипов, С.А. Кукушкин, Н.В. Веселов. ПЖТФ 37, 72 (2011).

[20] K. Hiramatsu. Effects of buffer layers and advanced technologies on heteroepitaxy of GaN. Advances in Crystal Growth Research / Eds K. Sato, Y. Furukawa, K. Nakajima. Elsevier Amsterdam-London-N. Y.-Oxford-Paris-ShannonTokyo (2001). P. 210.

[21] Р.С. Телятник, А.В. Осипов, С.А. Кукушкин. ФТТ 57, 153 (2015).

[22] J. Neugebauer, T. Zywietz, M. Scheer, J. Northrup. Appl. Surf. Sci. 159-160, 355 (2000).

[23] X. Gonze, B. Amadon, P.M. Anglade, J.-M. Beuken, F. Bottin, P. Boulanger, F. Bruneval, D. Caliste, R. Caracas, M. Cote, T. Deutsch, L. Genovese, Ph. Ghosez, M. Giantomassi, S. Goedecker, D. Hamann, P. Hermet, F. Jollet, G. Jomard, S. Leroux, M. Mancini, S. Mazevet, M.J.T. Oliveira, G. Onida, Y. Pouillon, T. Rangel, G.-M. Rignanese, D. Sangalli, R. Shaltaf, M. Torrent, M.J. Verstraete, G. Z'erah, J.W. Zwanziger. Comp. Phys. Commun. 180, 2582 (2009).

[24] Silicon Carbide / Eds W.J. Choyke, H.M. Matsunami, G. Pensl. Akademie Berlin (1998). V. 2.

[25] В.Г. Дубровский. Теория формирования эпитаксиальных наноструктур. Физматлит, М. (2009). 351 с.

[26] С.А. Кукушкин, А.В. Осипов, А.В. Редьков. ФТТ 56, 2440 (2014).

[27] Y. Kumagai, K. Takemoto, J. Kikuchi, T. Hasegawa, H. Murakami, A. Koukitu. Phys. Status Solidi (b) 243, 1431 (2006).

[28] A. Koukitu, S. Hama, T. Taki, H. Seki. Jpn. J. Appl. Phys. 37, 762 (1998).

[29] А.А. Барыбин. Электроника и микроэлектроника. Физикотехнологические основы. Физматлит. М. (2006). 424 с.

[30] С.А. Кукушкин, А.В. Осипов. УФН 168, 1083 (1998). 